Electronic Supporting Information

\title{
Enhanced Surface and Bulk Recombination Kinetics by Virtue of Sequential Metal and Nonmetal Incorporation in Hematite-Based Photoanode for Superior Photoelectrochemical Water Oxidation
}

Tushar Kanta Sahu, ${ }^{\mathrm{a}}$ Adit Kumar Shah, ${ }^{\mathrm{a}}$ Avishek Banik ${ }^{\mathrm{a}}$ and Mohammad Qureshi ${ }^{\mathrm{a} *}$

a Department of Chemistry, Indian Institute Technology, Guwahati - 781039, Assam, India.

E-mail: mq@iitg.ernet.in; Fax: +91-361-2582349; Tel: +91-361-2582320
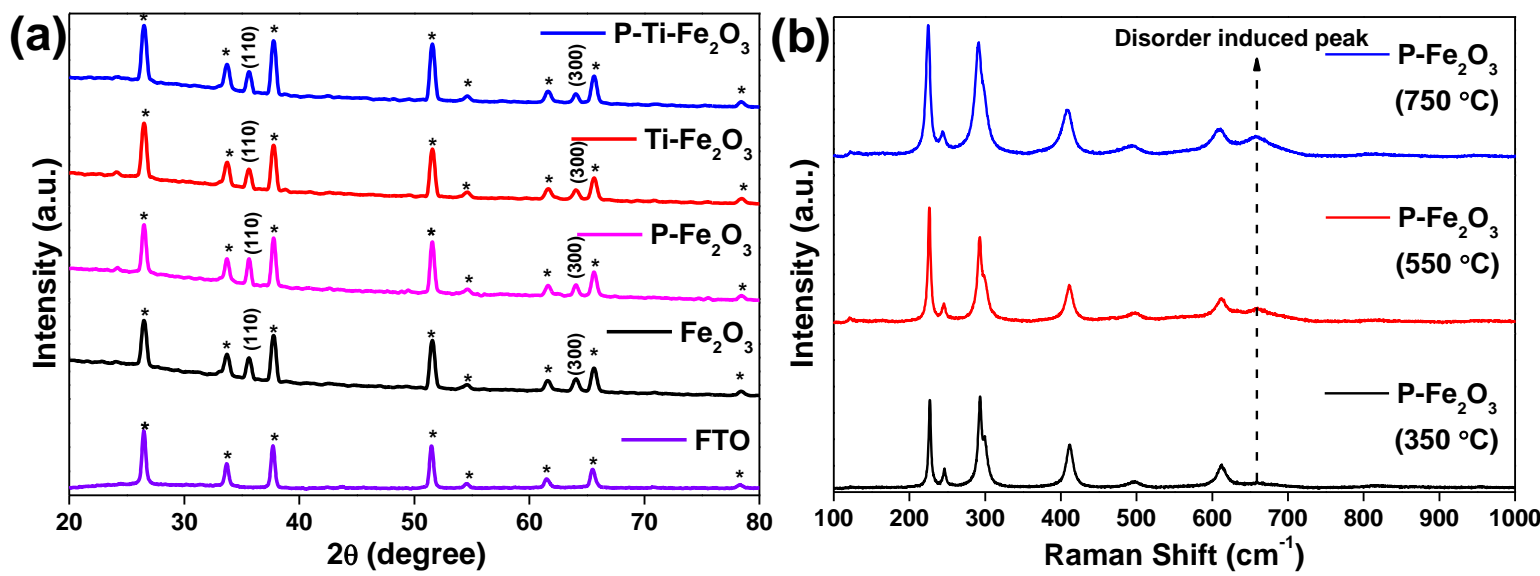

Fig. S1 (a) Powder XRD of all the photoanodes and (b) Raman spectra of P-incorporated over $\alpha-\mathrm{Fe}_{2} \mathrm{O}_{3}$ calcined at different temperatures. 


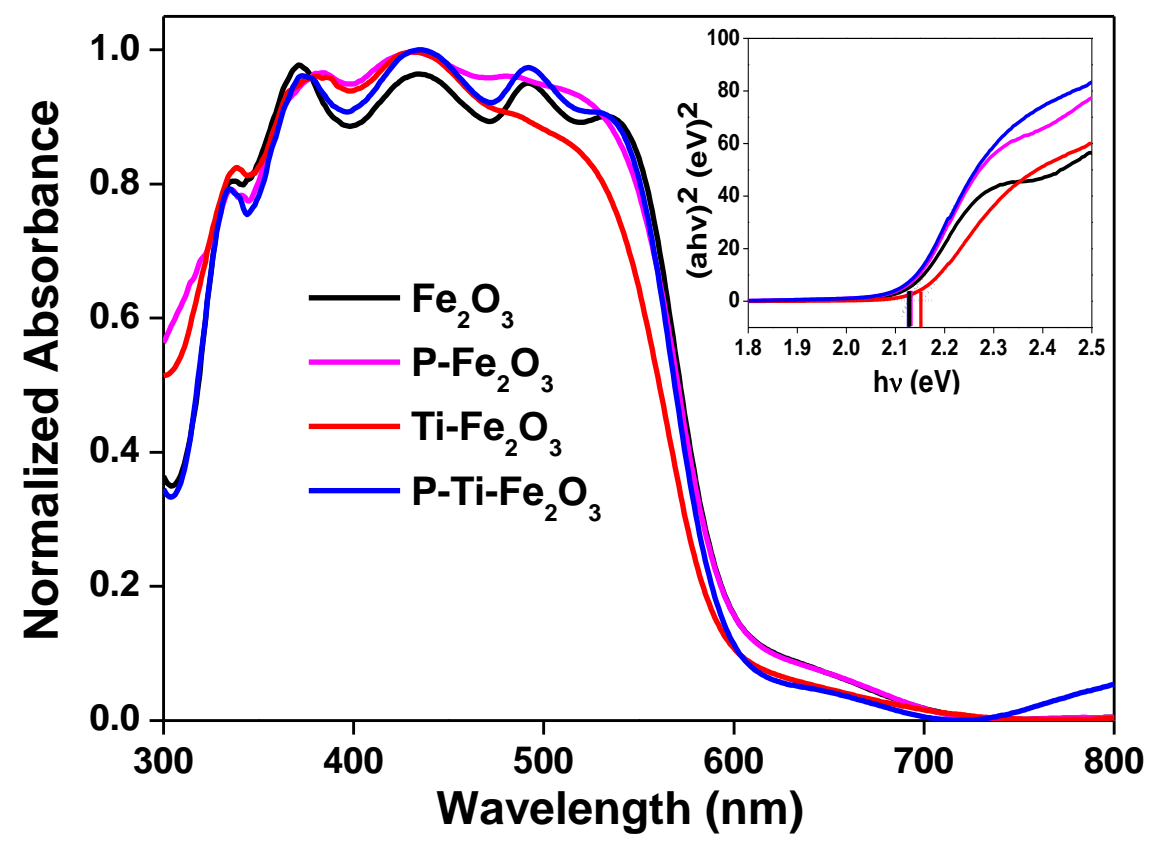

Fig S2. UV-Visible diffuse reflection spectra of $\alpha-\mathrm{Fe}_{2} \mathrm{O}_{3}, \mathrm{P}-\mathrm{Fe}_{2} \mathrm{O}_{3}, \mathrm{Ti}-\mathrm{Fe}_{2} \mathrm{O}_{3}$ and $\mathrm{P}-\mathrm{Ti}-\mathrm{Fe}_{2} \mathrm{O}_{3}$. Inset showing Tauc's plots for all the photoanodes.
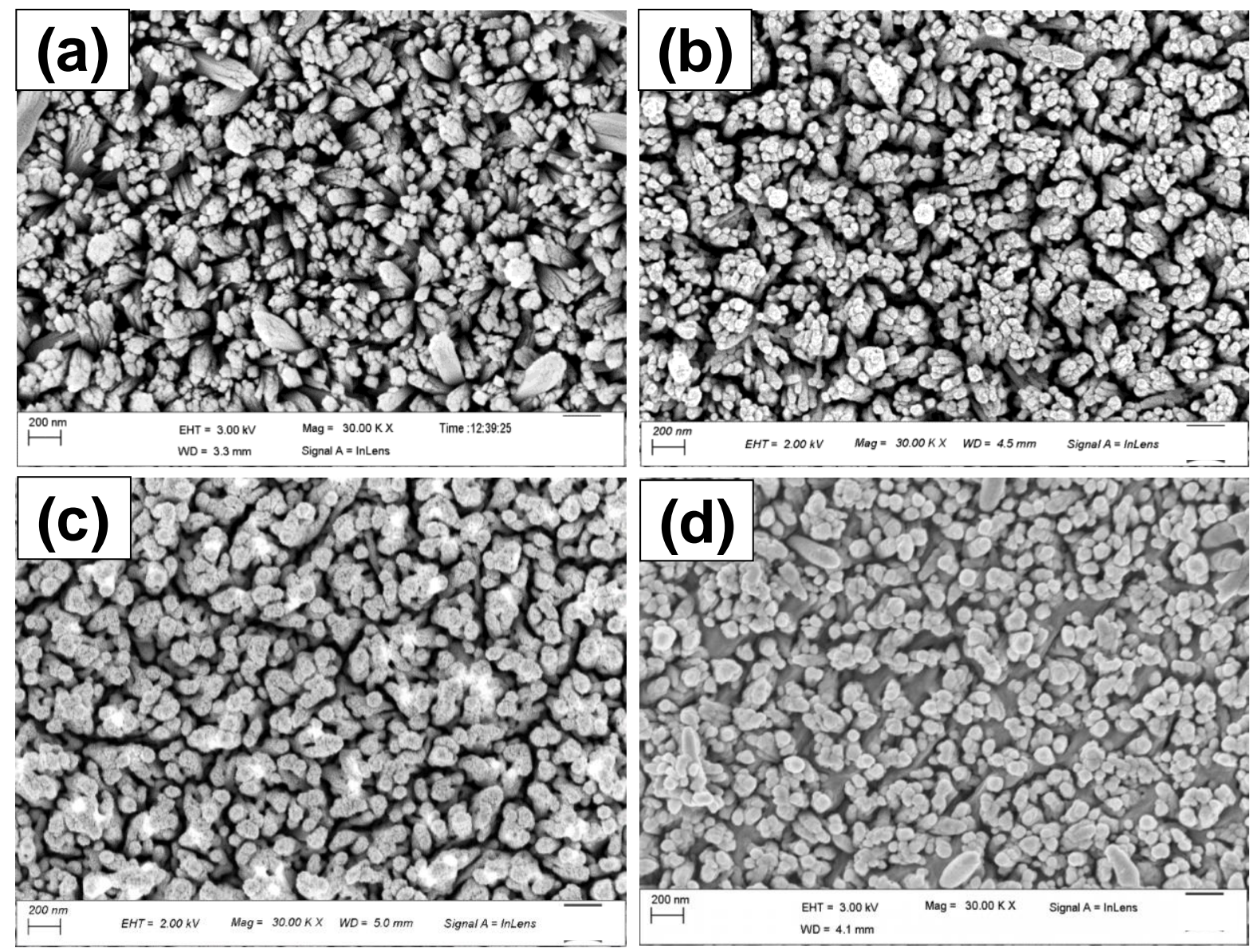

Fig. S3 FESEM images of (a) $\alpha-\mathrm{Fe}_{2} \mathrm{O}_{3}$, (b) $\mathrm{P}-\mathrm{Fe}_{2} \mathrm{O}_{3}$, (c) $\mathrm{Ti}-\mathrm{Fe}_{2} \mathrm{O}_{3}$ and (d) $\mathrm{P}-\mathrm{Ti}-\mathrm{Fe}_{2} \mathrm{O}_{3}$. 

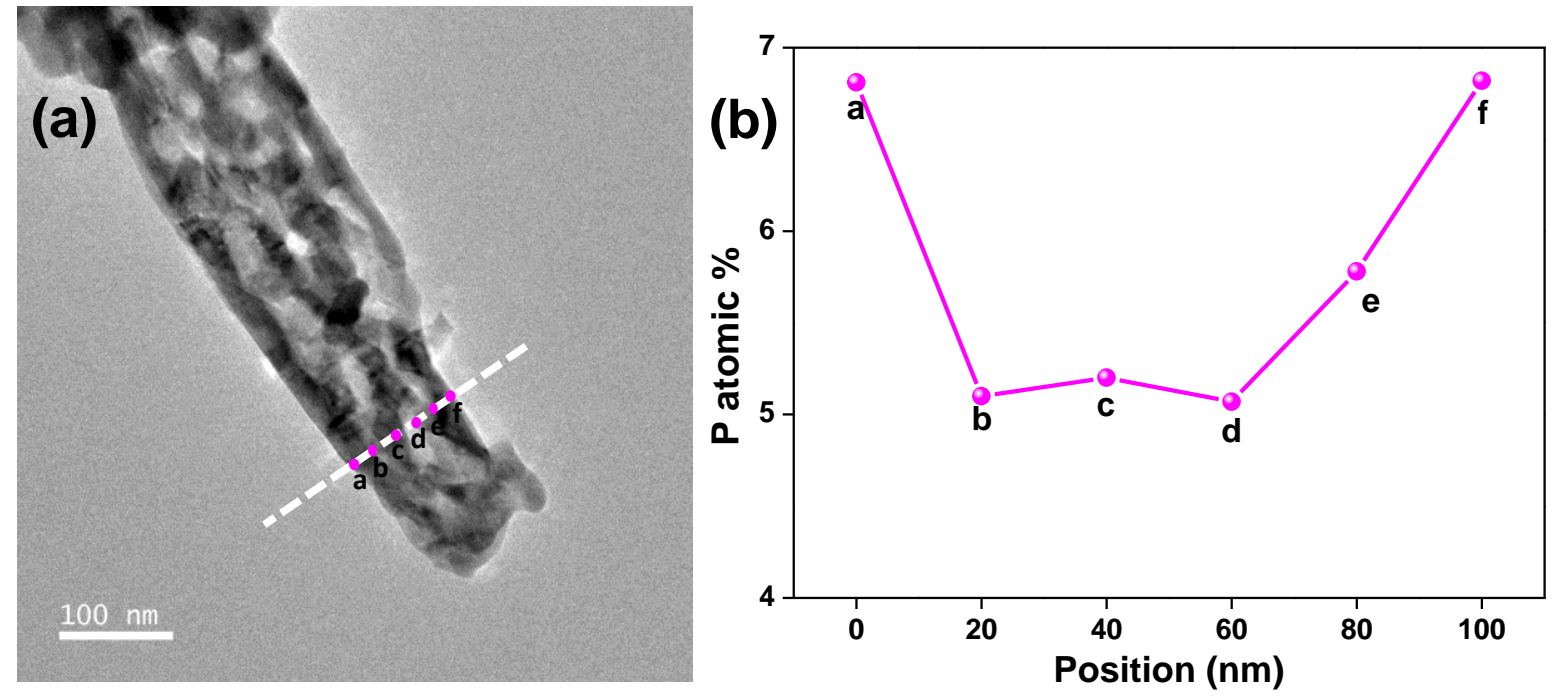

Fig. S4 (a) FETEM image of P-Ti- $\mathrm{Fe}_{2} \mathrm{O}_{3}$ and (b) phosphorus element concentration in a P-Ti$\mathrm{Fe}_{2} \mathrm{O}_{3}$ nanorod from black dots in image (a).

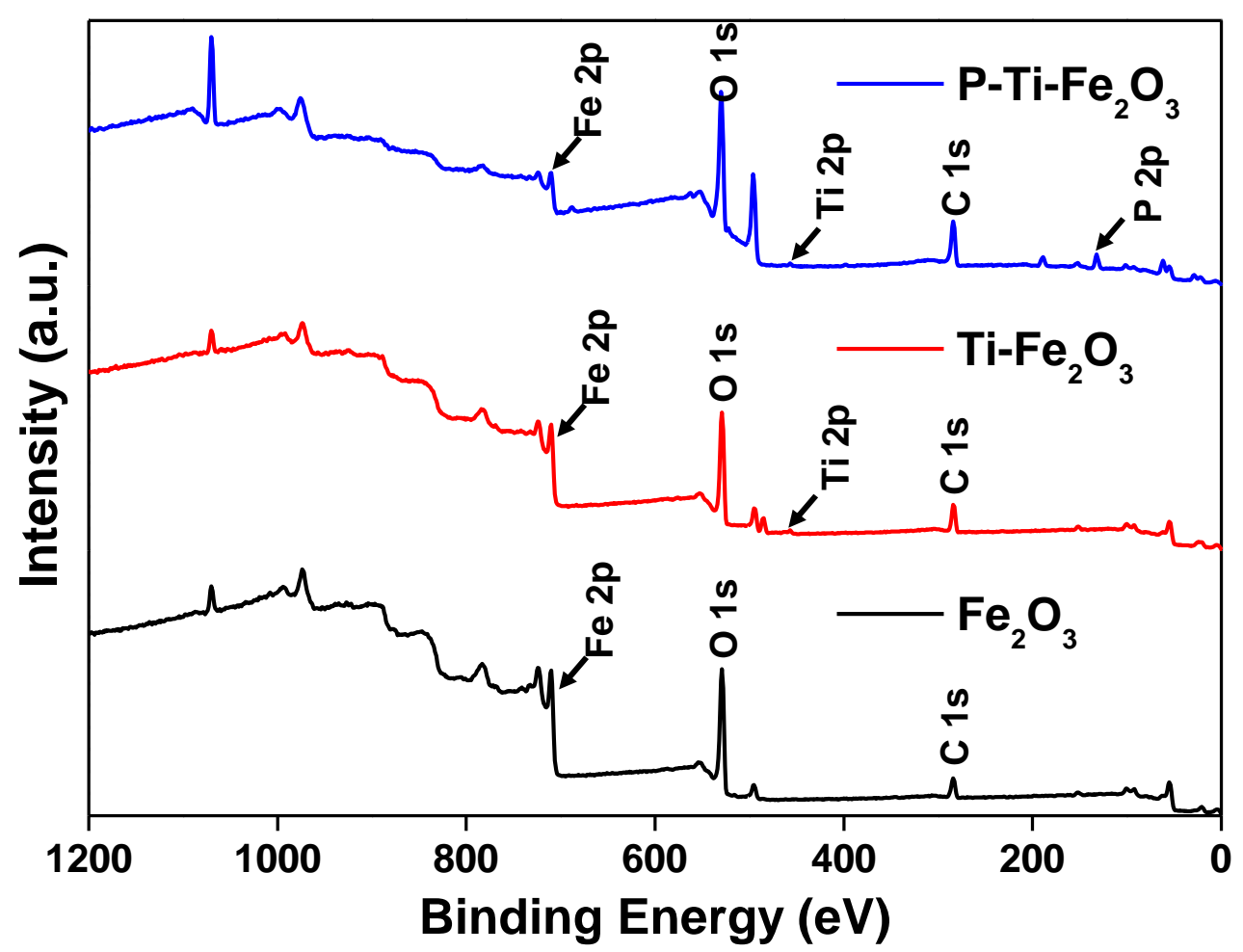

Fig. S5 XPS survey spectra of $\alpha-\mathrm{Fe}_{2} \mathrm{O}_{3}, \mathrm{Ti}-\mathrm{Fe}_{2} \mathrm{O}_{3}$ and $\mathrm{P}-\mathrm{Ti}-\mathrm{Fe}_{2} \mathrm{O}_{3}$. 

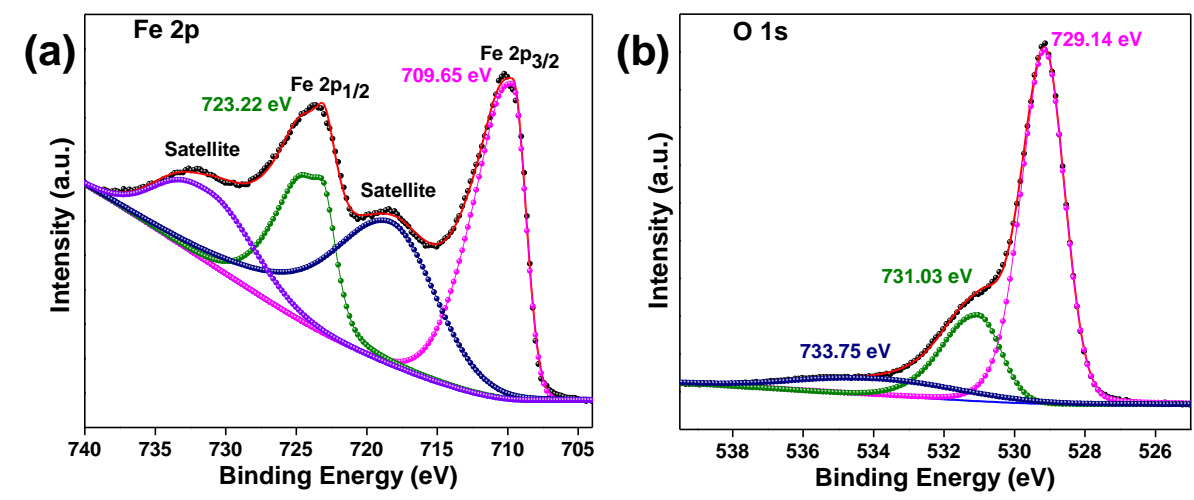

Fig. S6 XPS spectra of (a) Fe 2p core level and (b) O 1s core level of $\mathrm{Fe}_{2} \mathrm{O}_{3}$.
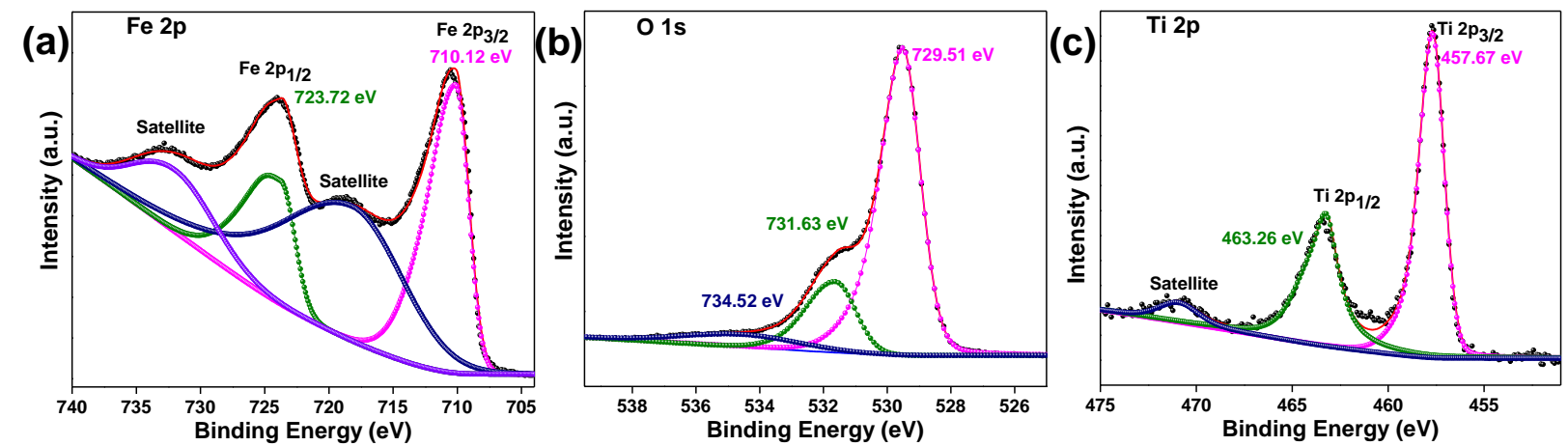

Fig. S7 XPS spectra of (a) Fe 2p core level, (b) O 1s core level and (c) Ti 2p core level of Ti$\mathrm{Fe}_{2} \mathrm{O}_{3}$.
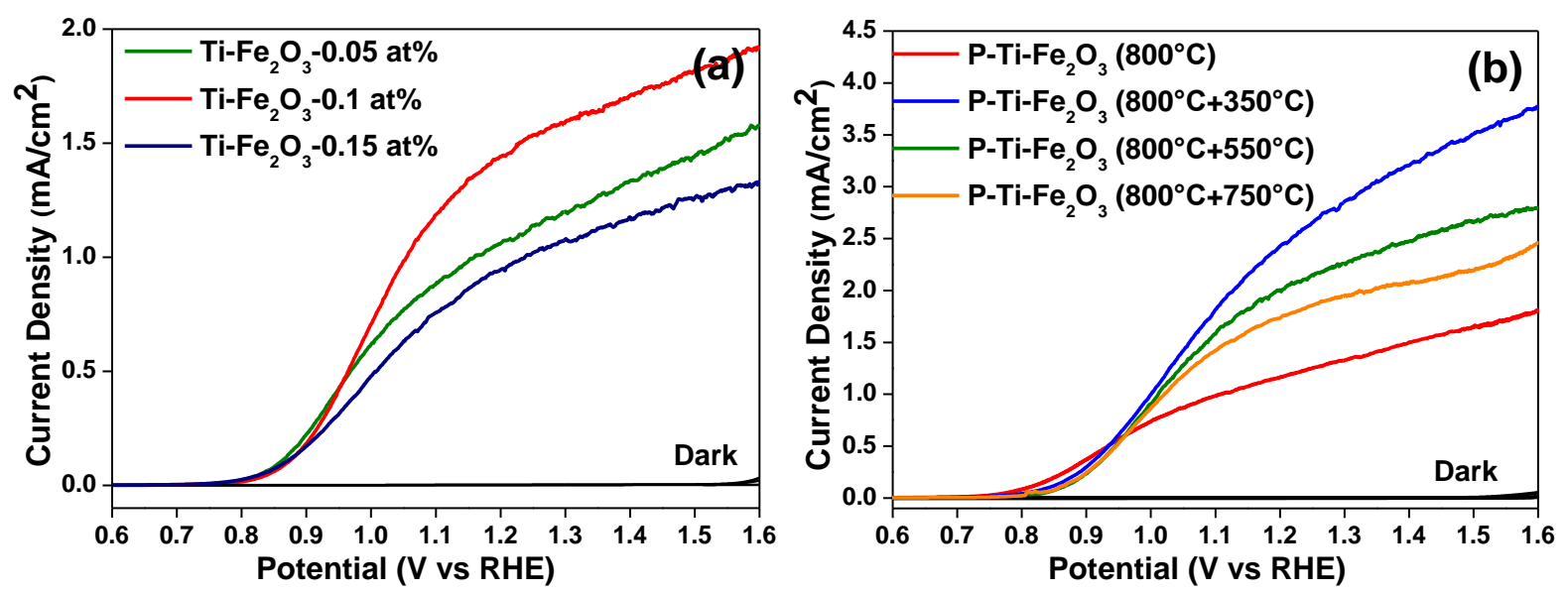

Fig. S8 (a) J-V characteristics of Ti-doped $\mathrm{Fe}_{2} \mathrm{O}_{3}$ photoanodes with different Ti content. (Fe:Ti $=0.05$ to 0.15 at \%) and (b) $\mathrm{J}-\mathrm{V}$ characteristics of P-incorporation with Ti-doped $\mathrm{Fe}_{2} \mathrm{O}_{3}$ with different annealing temperatures. 


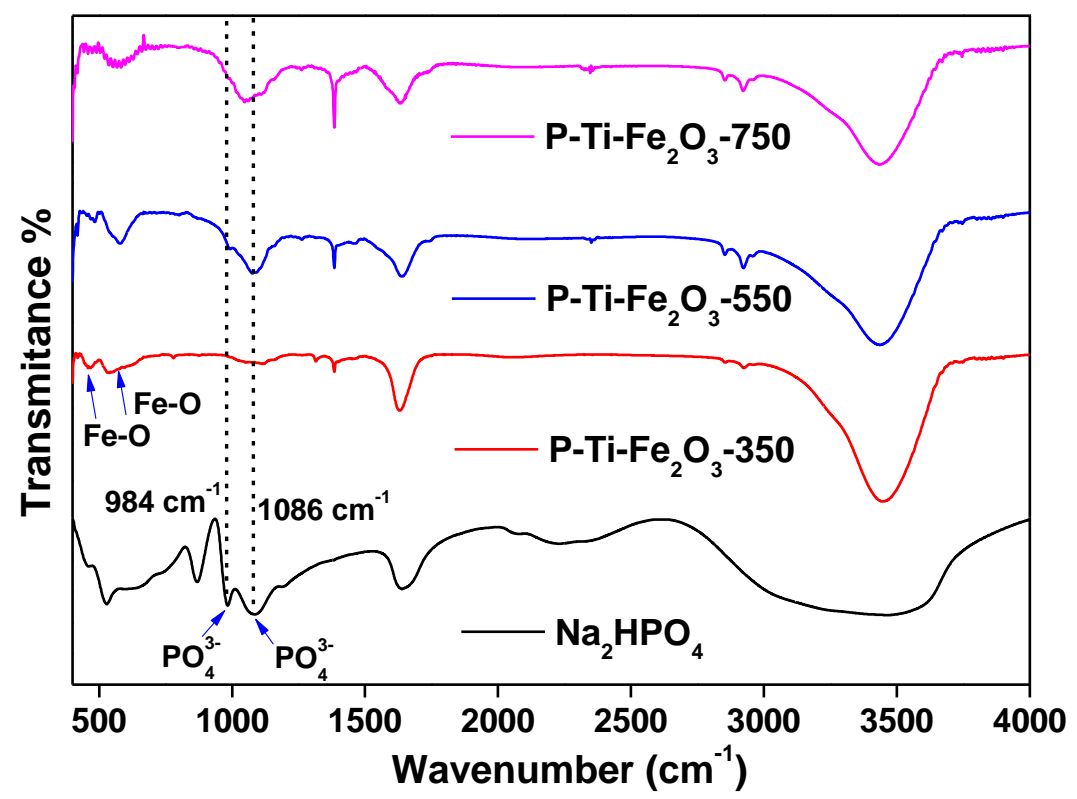

Fig. S9 FT-IR spectra of $\mathrm{Na}_{2} \mathrm{HPO}_{4}$ and $\mathrm{P}-\mathrm{Ti}-\mathrm{Fe}_{2} \mathrm{O}_{3}$ photoanodes calcined at different temperatures

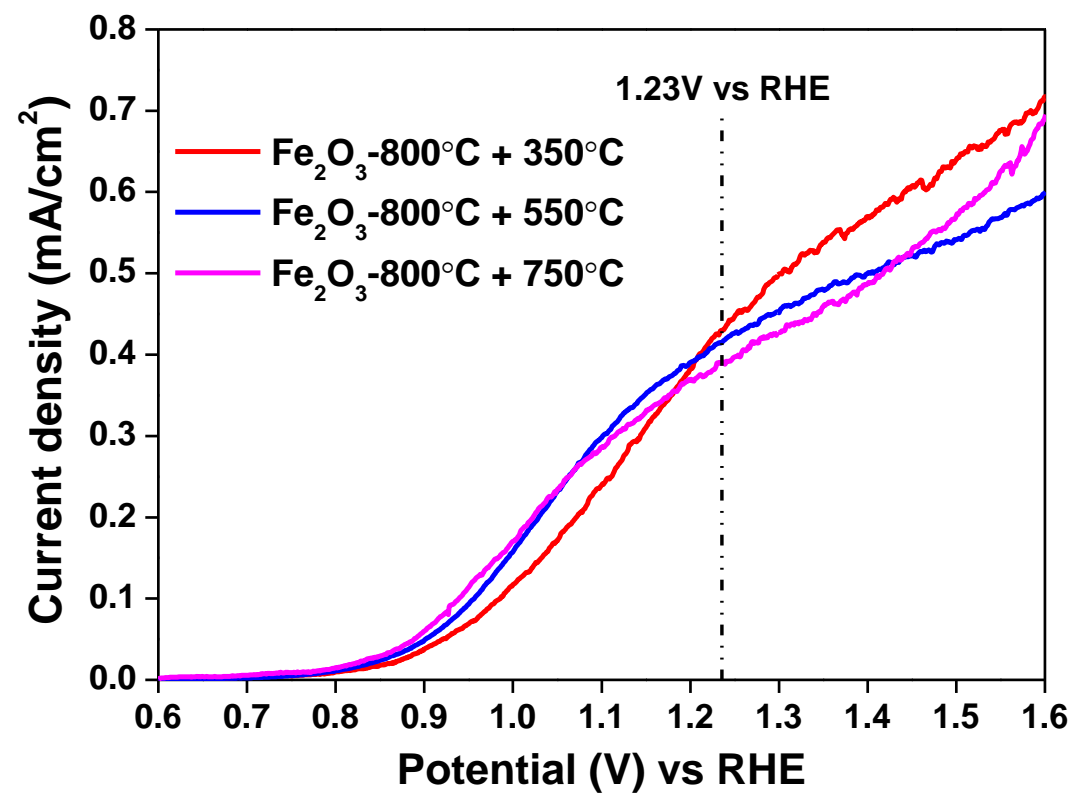

Fig. S10 Current density (J) vs. applied voltage (V) curves of bare $\mathrm{Fe}_{2} \mathrm{O}_{3}$ photoanodes calcined with two-step process. 


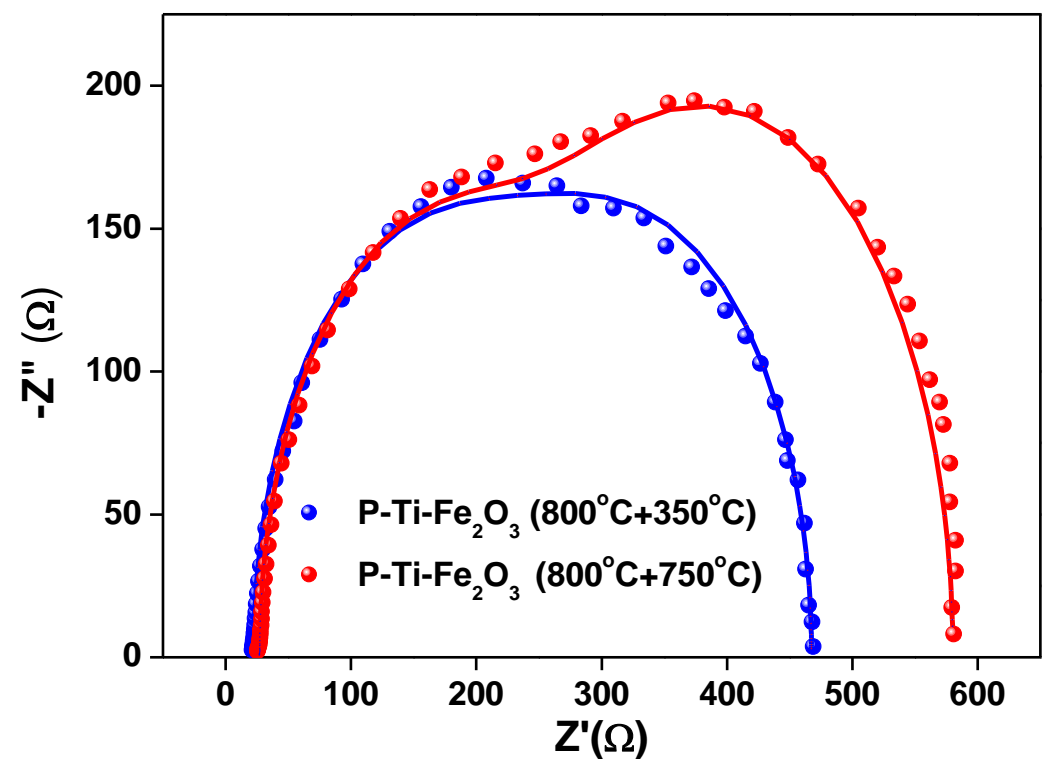

Fig. S11 The Nyquist plots of Ti and $\mathrm{P}$ co-modified $\alpha-\mathrm{Fe}_{2} \mathrm{O}_{3}$ with different calcination temperature with fittings under light illumination.
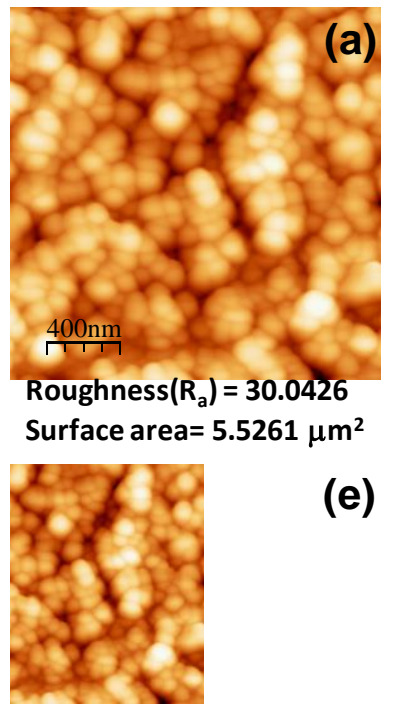

(e)
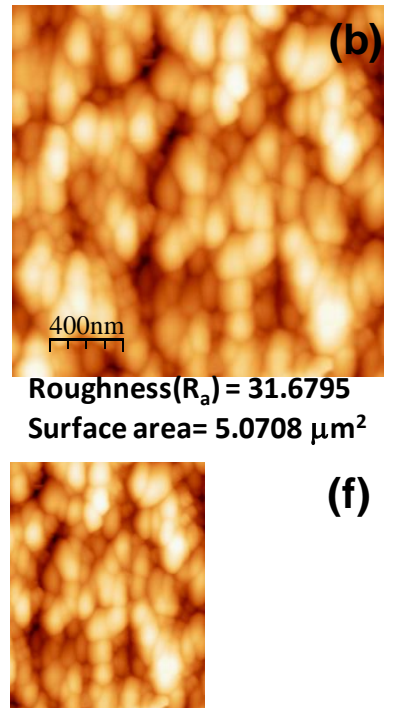

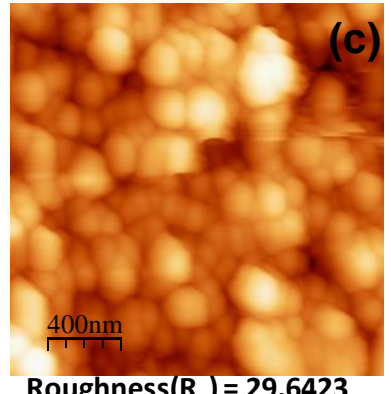

Roughness $\left(R_{\mathrm{a}}\right)=29.6423$ Surface area $=4.7411 \mu \mathrm{m}^{2}$

(f)

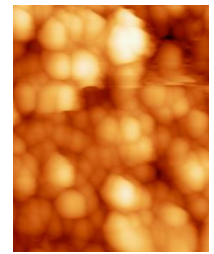

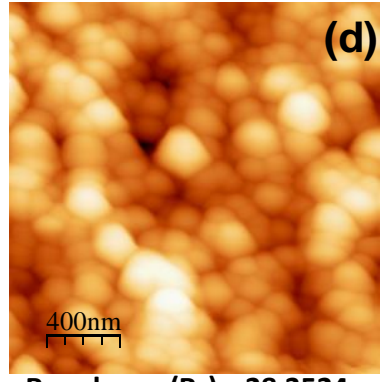

Roughness $\left(R_{\mathrm{a}}\right)=\mathbf{2 8 . 3 5 2 4}$

Surface area $=4.6878 \mu \mathrm{m}^{2}$

(g)

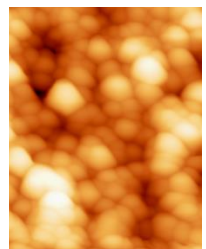

(h)

Fig. S12 AFM images of (a,e) $\mathrm{Fe}_{2} \mathrm{O}_{3}$, (b,f) P-Fe $\mathrm{F}_{2} \mathrm{O}_{3},(\mathrm{c}, \mathrm{g}) \mathrm{Ti}-\mathrm{Fe}_{2} \mathrm{O}_{3}$ and (d,h) P-Ti-Fe $\mathrm{O}_{3}$. 

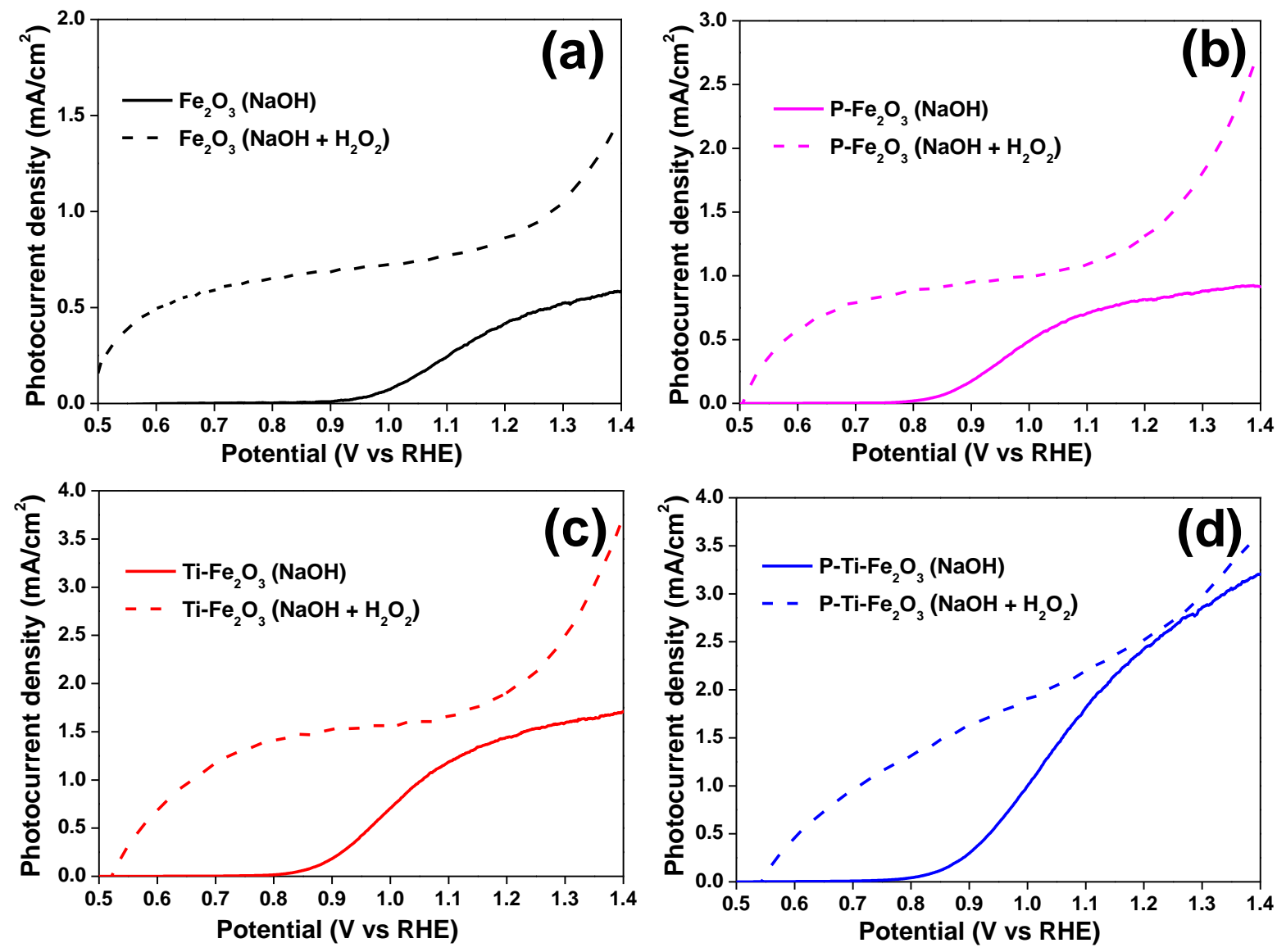

Fig. S13 Current density (J) vs. applied voltage (V) curves of (a) $\mathrm{Fe}_{2} \mathrm{O}_{3}$, (b) $\mathrm{P}_{-}-\mathrm{Fe}_{2} \mathrm{O}_{3}$, (c) Ti$\mathrm{Fe}_{2} \mathrm{O}_{3}$ and (d) $\mathrm{P}-\mathrm{Ti}-\mathrm{Fe}_{2} \mathrm{O}_{3}$ photoanodes under light illumination at a scan rate of $10 \mathrm{mV} / \mathrm{s}$ in presence of hole-scavenger $\mathrm{H}_{2} \mathrm{O}_{2}$.

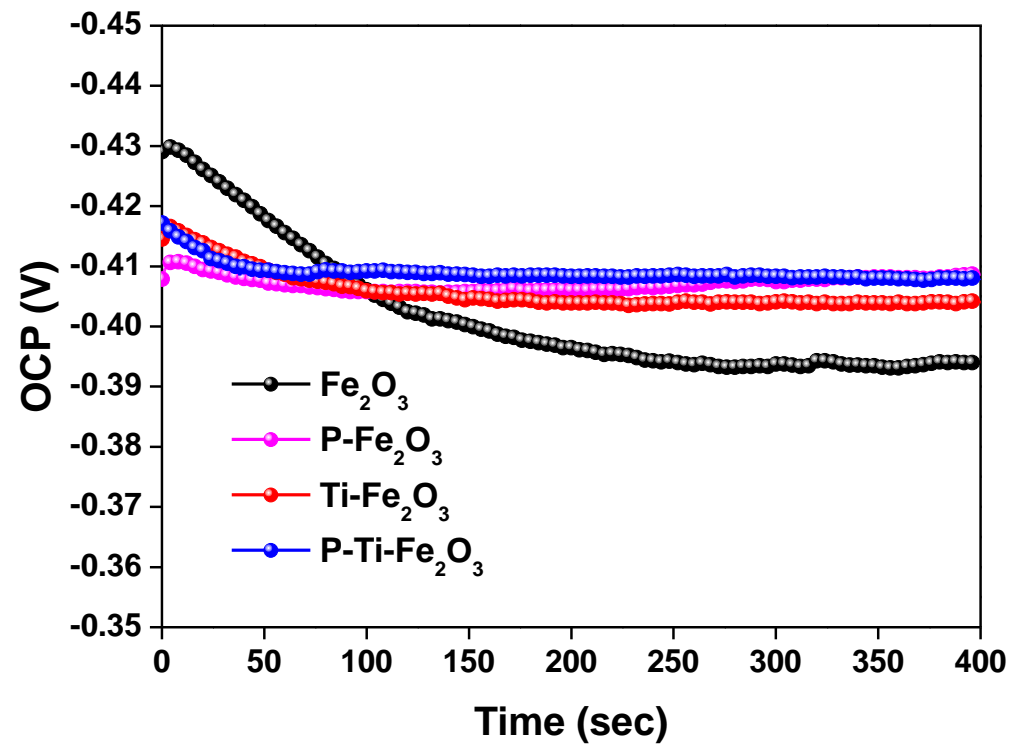

Fig. S14 Open circuit potential (OCP) of all the photoanodes under light illumination. 
Table S1 Fitting results of the EIS of Ti and P co-modified $\alpha-\mathrm{Fe}_{2} \mathrm{O}_{3}$ with different calcination temperature.

\begin{tabular}{|c|c|c|c|c|c|}
\hline Photoanode & $\mathbf{R}_{\mathrm{s}} / \mathbf{\Omega}$ & $\mathbf{R}_{\text {trap }} / \mathbf{\Omega}$ & $10^{-6} \times C_{\text {bulk }} / F$ & $\mathrm{R}_{\mathrm{ct}} / \Omega$ & $10^{-5} \times \mathrm{C}_{\text {trap }} / \mathrm{F}$ \\
\hline $\begin{array}{l}\text { P-Ti-Fe } 2 \mathrm{O}_{3} \\
\left(800^{\circ} \mathrm{C}+750^{\circ} \mathrm{C}\right)\end{array}$ & 27.08 & 307.8 & 22.52 & 245.3 & 17.38 \\
\hline $\begin{array}{l}\mathrm{P}-\mathrm{Ti}-\mathrm{Fe}_{2} \mathrm{O}_{3} \\
\left(800^{\circ} \mathrm{C}+350^{\circ} \mathrm{C}\right)\end{array}$ & 22.34 & 298.4 & 13.11 & 146.7 & 10.74 \\
\hline
\end{tabular}

Table S2 Comparison with previous reports based on metal and non-metal doped hematite.

\begin{tabular}{|c|c|c|c|}
\hline Photoanodes & $\begin{array}{l}\text { Synthetic } \\
\text { protocol }\end{array}$ & $\begin{array}{l}\text { Current density } \\
\text { at } 1.23 \mathrm{~V} \text { vs } \\
\text { RHE }\left(\mathrm{mA} / \mathrm{cm}^{2}\right)\end{array}$ & Reference \\
\hline Ti-doped $\mathrm{Fe}_{2} \mathrm{O}_{3}$ & Hydrothermal & $\begin{array}{l}0.66 \mathrm{~mA} / \mathrm{cm}^{2} \text { at } \\
1.0 \mathrm{~V} \text { vs RHE }\end{array}$ & [1] \\
\hline Ti-doped $\mathrm{Fe}_{2} \mathrm{O}_{3}$ & Hydrothermal & $1.91 \mathrm{~mA} / \mathrm{cm}^{2}$ & [2] \\
\hline $\mathrm{P}-\mathrm{Fe}_{2} \mathrm{O}_{3}$ & Hydrothermal & $2.7 \mathrm{~mA} / \mathrm{cm}^{2}$ & [3] \\
\hline $\begin{array}{l}\text { (Co, } \mathrm{Sn}) \text { co-doped } \\
\mathrm{Fe}_{2} \mathrm{O}_{3}\end{array}$ & Hydrothermal & $1.25 \mathrm{~mA} / \mathrm{cm}^{2}$ & [4] \\
\hline $\begin{array}{l}\text { (Sn, Be) co-doped } \\
\mathrm{Fe}_{2} \mathrm{O}_{3}\end{array}$ & Hydrothermal & $1.7 \mathrm{~mA} / \mathrm{cm}^{2}$ & [5] \\
\hline $\begin{array}{l}(\mathrm{Sn}, \mathrm{Zr}) \text { co-doped } \\
\mathrm{Fe}_{2} \mathrm{O}_{3}\end{array}$ & Hydrothermal & $1.64 \mathrm{~mA} / \mathrm{cm}^{2}$ & [6] \\
\hline $\begin{array}{l}\mathrm{Sn}, \mathrm{Zr}-\mathrm{Fe}_{2} \mathrm{O}_{3}- \\
\mathrm{NiOOH}\end{array}$ & Hydrothermal & $1.64 \mathrm{~mA} / \mathrm{cm}^{2}$ & [7] \\
\hline $\mathrm{MnO}_{2} / \mathrm{P}: \mathrm{Fe}_{2} \mathrm{O}_{3}$ & Hydrothermal & $1.65 \mathrm{~mA} / \mathrm{cm}^{2}$ & [8] \\
\hline $\mathrm{NiO} / \mathrm{P}-\mathrm{Fe}_{2} \mathrm{O}_{3}$ & Hydrothermal & $2.08 \mathrm{~mA} / \mathrm{cm}^{2}$ & [9] \\
\hline $\mathrm{P}-\mathrm{Fe}_{2} \mathrm{O}_{3} / \mathrm{Ce}-\mathrm{Pi}$ & Hydrothermal & $1.24 \mathrm{~mA} / \mathrm{cm}^{2}$ & [10] \\
\hline $\mathrm{CoPi} / \mathrm{P}-\mathrm{Fe}_{2} \mathrm{O}_{3}$ & Hydrothermal & $0.89 \mathrm{~mA} / \mathrm{cm}^{2}$ & [11] \\
\hline Sn-doped $\mathrm{Fe}_{2} \mathrm{O}_{3}$ & Hydrothermal & $1.24 \mathrm{~mA} / \mathrm{cm}^{2}$ & [12] \\
\hline $\mathrm{P}-\mathrm{Ti}-\mathrm{Fe}_{2} \mathrm{O}_{3}$ & Hydrothermal & $2.56 \mathrm{~mA} / \mathrm{cm}^{2}$ & This Work \\
\hline
\end{tabular}

References-

1. Shen, S. H.; Kronawitter, C. X.; Wheeler, D. A.; Guo, P. H.; Lindley, S. A.; Jiang, J. G.; Zhang, J. Z.; Guo, L. J.; Mao, S. S. Physical and Photoelectrochemical Characterization of Ti-doped Hematite Photoanodes Prepared by Solution Growth $J$. Mater. Chem. A 2013, 1, 14498- 14506 DOI: 10.1039/c3ta13453a 
2. Deng, J.; Zhong, J.; Pu, A.; Zhang, D.; Li, M.; Sun X.; Lee, S. T. Ti-doped Hematite Nanostructures for Solar Water Splitting with High Efficiency. J. Appl. Phys. 2012, 112, 084312, DOI: 10.1063/1.4759278

3. Zhang, Y.; Jiang, S.; Song, W.; Zhou, P.; Ji, H.; Ma, W.; Hao, W.; Chen, C.; Zhao , J. Nonmetal P-doped Hematite Photoanode with Enhanced Electron Mobility and High Water Oxidation Activity. Energy Environ. Sci. 2015, 8, 1231-1236, DOI: 10.1039/C4EE03803G.

4. Wang, J.; Du, C.; Peng, Q.; Yang, J.; Wen, Y.; Shan B.; Chen, R. Enhanced Photoelectrochemical Water Splitting Performance of Hematite Nanorods by Co and Sn co-doping. Int. J. Hydrogen Energy 2017, 42, 29140-29149, DOI: 10.1016/j.ijhydene.2017.10.080

5. Annamalai, A.; Lee, H. $\quad$ H.; Choi, S. $\quad$ H.; Lee, S. $\quad$ Y.; GraciaEspino, E.; Subramanian, A.; Park, J.; Kong, K.-J.; Jang, J. S. Sn/Be Sequentially co-doped Hematite Photoanodes for Enhanced Photoelectrochemical Water Oxidation: Effect of $\mathrm{Be}^{2+}$ as co-dopant Sci. Rep. 2016, 6, 23183 DOI: $10.1038 /$ srep23183

6. Chen, D.; Liu, Z. Dual-Axial Gradient Doping (Zr and Sn) on Hematite for Promoting Charge Separation in Photoelectrochemical Water Splitting. ChemSusChem 2018, 11, 3438-3448, DOI: $10.1002 /$ cssc. 201801614

7. Tamirat, A. $\quad$ G.; Su, W.-N.; Dubale, A. $\quad$ A.; Chen, H.-M.; Hwang, B.J. Photoelectrochemical water splitting at low applied potential using a $\mathrm{NiOOH}$ coated codoped ( $\mathrm{Sn}, \mathrm{Zr}) \alpha-\mathrm{Fe}_{2} \mathrm{O}_{3}$ photoanode. J. Mater. Chem. A 2015, 3, 59495961, DOI: 10.1039/C4TA06915C

8. Rui, Q.; Wang, L.; Zhang, Y.; Feng, C.; Zhang, B.; Fu, S.; Guo, H.; Hu, H.; Bi, Y. Synergistic Effects of P-doping and a $\mathrm{MnO}_{2}$ Cocatalyst on $\mathrm{Fe}_{2} \mathrm{O}_{3}$ Nanorod Photoanodes for Efficient Solar Water Splitting. J. Mater. Chem. A 2018, 6, 70217026, DOI: 10.1039/C8TA00556G

9. Li, F.; Li, J.; Zhang, J.; Gao, L.; Long, X.; Hu, Y.; Li, S.; Jin, J.; Ma, J. NiO Nanoparticles Anchored on Phosphorus-Doped $\alpha-\mathrm{Fe}_{2} \mathrm{O}_{3}$ Nanoarrays: An Efficient Hole Extraction p-n Heterojunction Photoanode for Water Oxidation. ChemSusChem 2018, 11, 2156-2164, DOI: 10.1002/cssc.201800571

10. Bu, X.; Gao, Y.; Zhang S.; Tian, Y. Amorphous Cerium Phosphate on P-doped $\mathrm{Fe}_{2} \mathrm{O}_{3}$ Nanosheets for Efficient Photoelectrochemical Water Oxidation. Chem. Eng. J. 2019, 355, 910-919, DOI: 10.1016/j.cej.2018.08.221 
11. Wu, D.; Zhang, Z. Simultaneous Non-metal Doping and Cocatalyst Decoration for Efficient Photoelectrochemical Water Splitting on Hematite Photoanodes Electrochim. Acta. 2018, 282, 48-55, DOI: 10.1016/j.electacta.2018.06.045

12. Ling, Y. C.; Wang, G. M.; Wheeler, D. A.; Zhang, J. Z.; Li, Y. Sn-Doped Hematite Nanostructures for Photoelectrochemical Water Splitting. Nano Lett. 2011, 11, 2119-2125, DOI: 10.1021/nl200708y 